\title{
Human Identity Verification by Using Physiological and Behavioural Biometric Traits
}

\author{
S. M. E. Hossain and G. Chetty
}

\begin{abstract}
Biometric authentication of a person is highly challenging and complex problem. A significant research effort has gone into this area and a number of research works were published, but still there is an immense shortage of accurate and robust methods and techniques. In this paper we survey several important research works published in this area and we found our new technology to identify a person using multimodal physiological and behavioural biometrics. For our first stage of experimental evaluation, we used side face and gait for our experiments and we achieved around $100 \%$ recognition rate.
\end{abstract}

Index Terms-Multimodal, next-generation, physiological biometric, robust-method

\section{INTRODUCTION}

Biometric person identification is a common technological tool for identity verification. It carries significant importance for national or international security. All most each and every part of human body is unique; some of the significant ones have been used for developing automated identity verification systems. Fingerprint, palm print, face, iris, ear [1], [2] etc. have been used immensely for current generation of person authentication technologies. There are still challenges in this area, and need for better biometric modalities, development of novel approaches and techniques are continually an ongoing process. Video surveillance in public places and facilities has become omnipresent, and has become the first line of defence for protecting assets and people for different types of operating scenarios and applications - be it a civilian public space for access control to a facility, or financial and transaction oriented applications, or the high security immigration and border control check points. It has become an enabler of trust, integrity and security in the new Digital Economy [3]. The need for non-intrusive biometric modalities enjoys significant user acceptability. Any one biometric modality on its own cannot address all the challenges, and importance of combining the information from multiple biometric modalities holds significant promise. Researchers started to work on multimodal biometrics and have discovered several new ways of combining them. However, most of the studies haven't considered the real world applicability and usability of these novel ideas. Recently, recognizing identity from gait patterns has become a popular area of research in biometrics and computer vision, and one of the most successful applications of image analysis and understanding. Gait

Manuscript received July 14, 2011; revised August 28, 2011.

Authors are with Faculty of information Science \& Engineering, University of Canberra, Australia (email: emdad.hossain@canberra.edu.au) recognition is one of the new and important biometric technologies based on behavioural characteristics, and it involves identifying individuals by their walking patterns. In the research work reported in this paper, we have investigated profile face (side face) and gait as novel biometric modalities for ascertaining the identity of the person under real world video surveillance scenarios.

\section{BACKGROUND}

Person authentication using fingerprint, face, iris, retina or voice biometric traits has increasingly being deployed for day-to day security and surveillance applications. However, one of most acceptable non-intrusive physiological attribute to authenticate is "face". Automated face recognition technology [4] first captured the public attention from the media reaction to a trial implementation at the January 2001 super bowl, which captured surveillance image and compared them to a database mugshots [4]. From 1960s till now vast number of research works has been conducted on biometric person authentication. Several research articles have been reported in use of signature, fingerprint, face and voice biometrics [5]. For face recognition systems, the performance of $2 \mathrm{D}$ face matching systems depends on capability of being insensitive of critical factors such as facial expression, makeup and aging, but also relies upon extrinsic factors such as illumination difference, camera viewpoint, and scene geometry [6]. In fact, none of the methods result in acceptable false error rates. However, most of the research focussed on attempts to achieve acceptable false error rates (around $1-5 \%$ ), if not perfect error rates.

Further, the 2D face recognition systems are vulnerable to pose, and illumination variations. Use of $3 \mathrm{D}$ face can make systems robust to pose and illumination variations. The state of the art 3D face recognition technique using isogeodesic stripes was proposed in [6], 3D face recognition from single image using single reference face shape was proposed in [2], where researchers proposed a novel method for $3 \mathrm{D}$ shape recovery of faces that exploits the similarity of faces. It also should mention that a number of limitations of $3 \mathrm{D}$ identification are high costs, limited availability of databases [7].

There have been several works reporting use of fingerprints for authenticating identity. A fingerprint is made of a number of ridges and valleys on the surface of the finger [8]. The uniqueness of a fingerprint can be determined by the pattern of ridges and furrows as well as the minutiae points. There are five basic fingerprint patterns: arch, tented arch, left loop, right loop and whorl. Loops make up $60 \%$ of all fingerprints, whorls account for $30 \%$, and arches for $10 \%$. 
Fingerprints are usually considered to be unique, with no two fingers having the exact same dermal ridge characteristics [8]. In fact, there has been a debate on how stable is the uniqueness of fingerprints? Further, due to increasing use of fingerprints for criminal identification, there have been cases of abuse. Hundreds of asylum seekers in Sweden and French tried to cut or burn their fingertips to evade identification by "Eurodac", and EU fingerprint ID for asylum seekers [9], likewise, a Chinese women arrested for illegal entry had altered her fingerprints through surgery (Dec 08) [9]. According to most researchers, Iris and retina are not changeable, but still not out of limitation. The fail to enrol (FTE) rate brings up another important problem. Not all users can use any given biometric system. People without hands cannot use fingerprint or hand-based systems. Visually impaired people have difficulties using iris or retina based techniques. As not all users are able to use a specific biometric system, the authentication system must be extended to handle users falling into the FTE category. This can make the resulting system more complicated, less secure or more expensive [10]. The authors in [10] clearly indentified undeniable limitations for biometric person authentication using fingerprint, iris and retina. Same might goes to person authentication using signature, some systems may also compare visual images of signatures, but the core of a signature biometric system is behavioral, i.e. how it is signed rather than visual, i.e. the image of the signature [10]. It means it has limitations for usage with persons with disability, and it can't be applied to authenticate for large population due to behavioral nature of the trait.

Another possible biometric trait is use of hand geometry. In large populations, hand geometry is not suitable for so-called one-to-many applications, in which a user is identified from his biometric without any other identification [11]. Some extreme biometric traits have also been proposed such as use of ear canal. Researchers found that one of the most promising techniques is use of multimodality or combination of biometric traits. Using principle component analysis (PCA) on combined image of ear and face, researchers in [6], [12] have found that multi-modal recognition results in significant improvement over either individual biometric. Since we have reviewed some of the prominent biometric traits for person authentication, we now look at some of the desirable characteristics of biometric traits as proposed by [1]. Next Section discusses some of the desirable characteristics for good biometric traits.

\section{A. Comparison of Various Biometric Technologies}

The choice of a particular human characteristic to be used as a biometric trait depends on the following criteria [13]:

1) Uniqueness is how well the biometric separates individually from another.

2) Permanence measures how well a biometric resists aging.

3) Collectability ease of acquisition for measurement.

4) Performance accuracy, speed, and robustness of technology used.

5) Acceptability degree of approval of a technology.

6) Circumvention ease of use of a substitute.

The following figure shows a comparison of existing biometric systems in terms of those parameters. A low ranking indicates poor performance in the evaluation criterion whereas a high ranking indicates a very good performance [13].

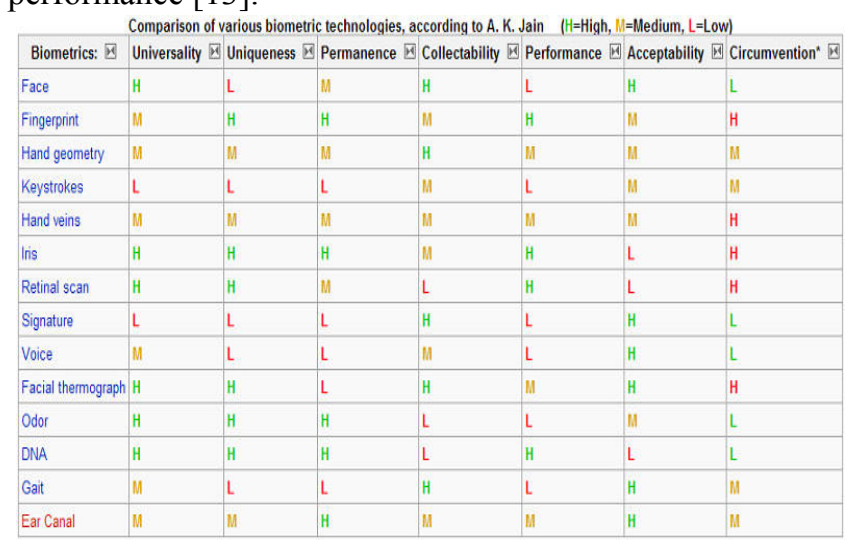

Fig. 1. Circumvent ability listed with reversed colours because low is desirable here instead of high [13]

As can be seen in this Table, each and every individual technology has limitation either in universality, uniqueness, permanence, collectability, or performance, acceptability, circumvention. Due to these limitations, no single biometric can provide a desired performance and the usage of multimodal biometric traits sounds promising. Exploiting information from multiple biometric sources or features improves the performance and also robustness of person authentication [14].One of most widely reported multimodal biometric authentication is combination of speech and signature features. Research shows that they result in good performance, but limited applications. Perhaps they didn't collect the data from practical environment [14]. So, that's still far from public applicability. Another popular multimodal trait is combined authentication of "face and iris", First of all face alone is not good enough to identify a personthat has been proved few times. Now, in the case of iris, there would be problem for disabled people, the research work reported by authors in [15] suggest usage of iris and face biometrics for robust identification and verification. They specifically applied 2-D discrete wavelet transform to extract the feature sets of low dimensionality from iris and face [15]. One interesting aspect of human iris is that a person iris might change if he/she undergoes a medical surgery on eye. Research shows it is possible to have colour surgery on human iris [16]. There has also been some work reported on fusion of face and ear biometric. However, the result obtained under controlled environment is about 4\% FRR (False Rejection Rate), and authors in [17] are working on improving the performance in uncontrolled operating environments. Next Section describes some of the futures directions in biometric identification technologies.

\section{NeXt Generation Biometric TeChNOLOGIES}

Having reviewed the capabilities and limitations of present current generation biometric identification technologies [1], [10], [14], [15], [17] and [18], we now discuss some of the next generation biometric technologies that could play a major role in security and authentication applications. 
According to authors in [1], the expectations of next generation identity verification involve addressing issues related to application requirements, user concern and integration. Some of the suggestions made to address these issues were use of non-intrusive biometric traits, role of soft biometrics or dominant primary and non-dominant secondary identifiers and importance of novel fusion protocols. Promising results from the preliminary investigations carried out in out lab, suggest significant role of gait biometrics in conjunction with face biometrics as potential candidates for next generation identity verification techniques. On $8^{\text {th }}$ March 2011, FBI (Federal Bureau of Investigation) of the United States has announced the capability of next generation biometric identification. According to their report, the single biometric traits like; fingerprint, iris, ear, face etc cannot play a vital role for next generation biometric identity verification.

The earliest biometric system that has been invented in 1882 was the "Bertillon System" [1]. That was a manual biometric authentication system and I can nominate the system as $1^{\text {st }}$ generation biometric identification system. From that till 1960's all most all the biometric authentication system was based on 'Bertillon System". In the early 1960's automated biometric identification have come into prominence in world security era. From 1960 till now, analysis and development has been progressing at the same pace. Number of large scale systems already been developed and implemented. This period I can nominate as $2^{\text {nd }}$ generation of biometric technology or current biometric technology. Even though, lot of good projects has been implemented successfully, there is still a need for better, robust, and publicly applicable biometric technologies to identify a person accurately. The need for better biometric identification trait is clarified by the world strongest law enforcement authority FBI (Federal Bureau of Investigation). It says; "the FBI Biometric Centre of Excellence (BCOE) will be leveraging the potential of newly emerging biometric technology to allow federal government agencies to increase their identity management capabilities. The $\mathrm{BCOE}$ will assist in implementing newly- developed biometric modalities such as facial recognition, iris recognition, and palm print matching into large-scale federal government biometric systems. Research will be performed to support the multimodal fusion of numerous biometrics to result in a significantly more accurate and comprehensive identity management system. The BCOE will also work on developing and enhancing other potential new biometric technologies including footprint and hand geometry, gait recognition, etc. [19]".

Single biometric trait, single biometric trait adoption with advance approaches and algorithms, and then multimodal biometric trait has been tested in several works. But today, researchers agree that, we have to have non-intrusive, physiological, and strong privacy protected multimodal biometric trait [20].

Most of the research works involved use of single biometric trait in a multimodal approach. Basically they used face to identify a person. Within the face they applied two modes, such as facial appearance and facial expression features [21]. This cannot be truly classified as the multimodal approach, may be a multi factor approach of single biometric trait. Another promising approach on use of multiple biometric traits is the fusion of face, ear and gait [22]. Researchers used Gabor and PCA features followed by decision level fusion. They used data set of 120 persons for three (3) different traits. And the best recognition performance that their proposed method achieved is $97.5 \%$. They used physiological biometric trait (frontal face, ear) that could have limitations on the application and implementation - because for real world video surveillance scenarios, it is hard to view the frontal face and ear at the same time. Here, a side face and gait could have been a more realistic and practical biometric data.

In this paper, we propose a fusion of side face - a physiological trait and the gait - a behavioral trait for automatic identity verification for low bandwidth video surveillance scenarios. Both the traits are absolutely non-intrusive, less vulnerable to forgery, and a strong privacy protected trait [20], since it is hard to copy someone's gait easily. Based on our preliminary experimental work involving feature level fusion of face and gait features on a publicly available low bandwidth video database, we can envisage the role of gait in conjunction with other biometrics such as face for next generation identification technology.

\section{A. Face-Gait Fusion models:}

We are currently investigating several novel algorithms and fusion models to integrate face, a physiological biometric, with gait, a behavioural biometric at low level and high level. Some of the work in progress are discussed here:

1) Face and gait feature fusion model: This new fusion approach will allow recognition of non-cooperating individuals at a distance in video, who expose side views to the camera. Information from two biometric sources, side face and gait, will be utilized and fused at feature level. For face, a high-resolution side face image will be constructed from multiple video frames. Same process will be followed for gait representation. Face features and gait features will be obtained separately using Principal Component Analysis (PCA) and Linier Discriminant Analysis (LDA) from the high-resolution side face image and gait image respectively. The system will be tested on a database of video sequences corresponding to several people. It is expected that this face-gait fusion approach will carry more discriminating power as compared to any individual biometric.

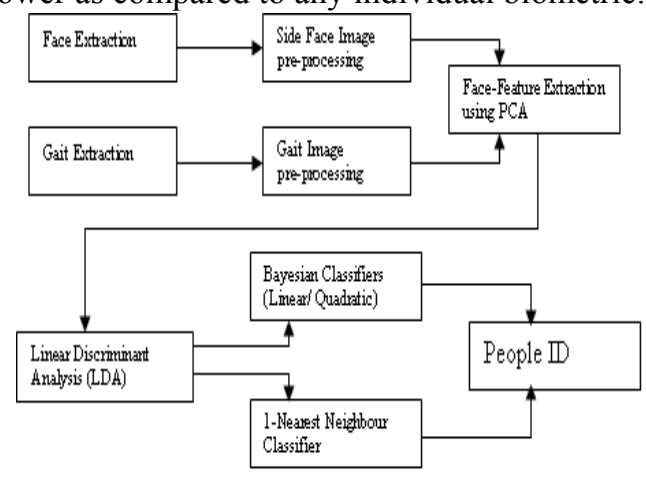

Fig. 2. Face and Gait Feature Fusion Model

The preliminary experiments of this approach have already 
been pursued in our lab, and the promising results from these experiments suggest this line of action to be yielding positive outcomes. For experimental evaluation of my proposed multimodal scheme, I used a publicly available video database of human actions [23]. This video database contains six types of human actions (walking, jogging, running, boxing, hand waving and hand clapping) performed several times by 25 subjects in four different scenarios: outdoors s1, outdoors with scale variation $\mathrm{s} 2$, outdoors with different clothes s3 and indoors s4. Currently the database contains 2391 sequences. All sequences were taken over homogeneous backgrounds with a static camera with $25 \mathrm{fps}$ frame rate. The sequences were down-sampled to the spatial resolution of $160 \times 120$ pixels and have a length of four seconds in average. We used only the walking sequences for my experiments and Figure 1 shows some of the sample images from the walking video sequences.

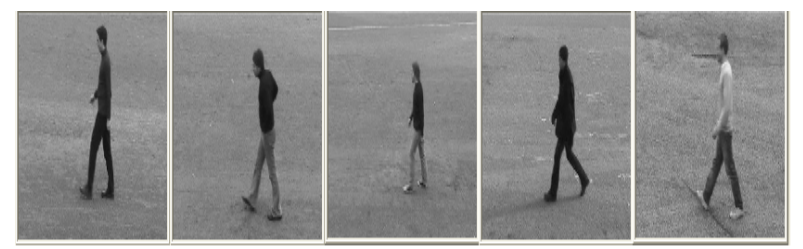

Fig. 3. Sample images from human action database for walking sequences [23].

For all the experiments, we used 100 video sequences for 25 people. There were 19 males and 6 females in the entire walking dataset. I performed some image pre-processing steps corresponding to cropping, filtering and histogram equalization and then extracted features based on PCA (principal component analysis) and LDA (linear discriminant analysis), the well known feature extraction and dimensionality reduction techniques. I used separate set for performing training and testing. The low dimensional PCA and LDA features were then classified by a Bayesian classifier. We tested with three different classifiers, the nearest neighbour $(k-N N)$, the Bayesian linear and the Bayesian quadratic classifiers. The combination of the low dimensional, discriminative PCA and LDA features along with powerful Bayesian classifiers allow us to achieve significant improvement in recognition accuracy as compared to conventional Euclidean distance based methods reported predominantly in previous works. This is because Bayesian classifiers have the flexibility to incorporate prior information, and can predict how a system's performance will change when going from one environment to another or when going from one type of testing to another [24]. Similarly, $k-N N$ is very effective simple classifier with noise reduction capabilities [25]. And our experimental results as follows:

TABLE 1: LDA FACE - GAIT FUSION WITH BAYESIAN CLASSIFIERS AND 1-NEAREST NEIGHBOUR CLASSIFIER

\begin{tabular}{|l|l|l|}
\hline Name & Partial Gait & Full-Gait \\
\hline k-NN Classify & $95 \%$ & $100 \%$ \\
\hline Bayesian Linear & $100 \%$ & $100 \%$ \\
\hline Bayesian Quadratic & $100 \%$ & $100 \%$ \\
\hline
\end{tabular}

2) Face and gait decision fusion model: For this approach, we are looking at Hidden Markov Models and Fisher faces method for gait and face classification, respectively. And then, the results obtained from the two classifiers will be utilized and integrated at match score level. The proposed face-gait fusion approach will be tested on video sequences of several individuals collected from different directions. The results of fusion of face and gait will be tested for robustness and better recognition performance compared with face only or gait-only method.

3) Static and dynamic body biometric decision fusion model: For this approach, a new human recognition algorithm by combining static and dynamic body biometrics is being investigated. For each sequence involving a walking human, temporal pose changes of the segmented moving silhouettes will be represented as an associated sequence of complex vector configurations and will then be analysed using the Procreates shape analysis method to obtain a compact appearance representation, called static information of body. In addition, a model-based approach under a condensation framework will be explored, which will track the walker and recover joint-angle trajectories of lower limbs, called dynamic information of gait. Both static and dynamic cues obtained from walking person video footage will be independently used for recognition using the nearest exemplar classifier. They will then be fused at the decision level using different combinations of rules and will be tested for improvement in performance for both identification and verification tasks. Experimental evaluation with a video surveillance dataset with several subjects (at least 20 - 30) will be done to demonstrate the feasibility of the proposed algorithm.

4) Multi camera cross-modal fusion model: For this approach, the face and gait cues will be derived from multiple simultaneous camera views, and we propose a visual hull algorithm for the fusion to create imagery in canonical pose prior to recognition. These view-normalized sequences, containing frontal images of face and profile silhouettes, will be separately used for face and gait recognition, and the results will be combined using a range of strategies. We will explore the concept of cross-modal correlation and score transformations for different modalities, with probabilistic settings for the cross-modal fusion. The effectiveness of various strategies will be evaluated on a data set with several subjects. We envisage that this novel fusion model will be useful in developing further statistical framework for multi-modal recognition.

5) Holistic and Hierarchical fusion protocols: For this fusion approach, we plan to investigate the important of a new fusion protocol, by integrating face and gait cues for the single camera case. We will employ a view invariant gait recognition algorithm for gait recognition. A sequential importance sampling based algorithm will be used for probabilistic face recognition from video. We will employ decision fusion to combine the results of our gait recognition algorithm and the face recognition algorithm. We then consider two new fusion protocols: hierarchical and holistic. The first protocol will involve 
using the gait recognition algorithm as a filter to pass on a smaller set of candidates to the face recognition algorithm. The second protocol will involve combining the similarity scores obtained individually from the face and gait recognition algorithms Simple rules like the SUM, MIN and PRODUCT will be used for combining the scores. The results of the fusion will be tested on a face-gait database which has outdoor gait and face data of several subjects.

6) Adaptive face-gait fusion model: For this fusion approach we plan to investigate adaptive fusion of face-gait patterns. Most work on information fusion for human identification is normally based on static fusion rules which cannot respond to the changes of the environment and the individual users. The adaptive fusion, which dynamically adjusts the fusion rules to suit the real-time external conditions. Two factors that may affect the relationship between gait and face in the fusion will be considered, i.e., the view angle and the subject-to-camera distance. Together they can determine the way gait and face are fused at an arbitrary time. Experimental evaluation will be carried out to assess the performance of adaptive fusion as compared to not only single biometric traits, but also those widely adopted static fusion rules including SUM, PRODUCT, MIN and MAX.

\section{B. Primary/Secondary identifier extraction:}

From the same face-gait video surveillance footage, high level contextual information or secondary identifiers such as gender, age, aggression and emotion will be extracted which can then be used to automatically enhance the confidence level and the reliability of the decision taken by human identification stage. The approach for gender recognition is described here. We propose a fusion model based on canonical correlation analysis (CCA) technique. The canonical correlation analysis (CCA) is a powerful multivariate statistical analysis tool, well suited for relating two sets of measurements, by fusing the two modalities at the feature level. Experiments on large datasets will be carried out to examine the gender recognition capability of face-gait fusion approach as compared to individual face and gait patterns. Figure 1 shows the proposed fusion approach fro gender (secondary identifier) extraction.

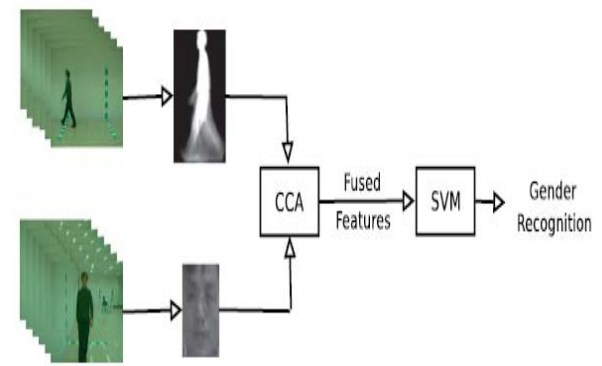

Fig. 4. Face-gait fusion for gender (secondary identifier) extraction

Using gait for determining gender is a novel approach, and has not been explored before. Most of the existing work attempts to classify gender from human faces. In our work, we would like to investigate structural features and dynamic features of gaits for gender recognition, by adopting Gait Energy Image (GEI), a novel spatiotemporal compact representation of gaits. GEI has been demonstrated to be effective for representing gaits in the human identification problem. Using background subtraction techniques, the walking subjects can be extracted from the original image sequences to derive binary silhouette image sequences.

To make the gait representation insensitive to the distance between the camera and the subject, we can perform silhouette pre-processing procedures including size normalization and horizontal alignment. Some examples of normalized and aligned silhouette images are shown in Figure 2. The entire human gait sequence can be divided into cycles as human walking repeats at a stable frequency. We decide the gait cycles by counting the number of foreground pixels in the bottom half of the silhouette and the two consecutive strides in the variation of the number constitute a gait cycle. Given the pre-processed binary silhouette image $B_{t}(x, y)$ at time $t$ in a sequence, the GEI is defined as follows:

$G(x, y)=\frac{1}{N} \sum_{t=1}^{N} B_{t}(x, y)$

where $\mathrm{N}$ is the number of frames in the complete cycle(s) of a silhouette sequence, $t$ is the frame number of the sequence, and $\mathrm{x}$ and $\mathrm{y}$ are values in the $2 \mathrm{D}$ image coordinate (see Figure 5 for an example of GEI). GEI reflects shapes of silhouette and their changes over the gait cycle, and it is not sensitive to incidental silhouette errors in individual frames [3].

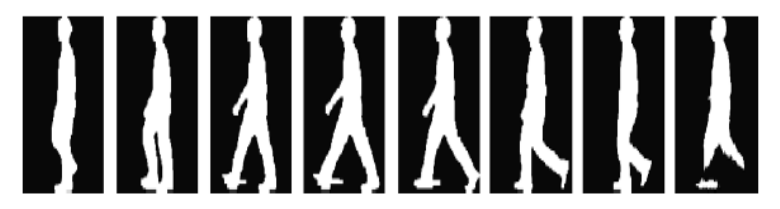

Fig. 5.Examples of normalized and aligned silhouette images. The rightmost image is the corresponding GEI.

\section{Protocols for fusion of primary and secondary biometric identifiers}

Once the primary identifiers and secondary identifiers are available, an appropriate protocol is needed to integrate the identifiers to address different user requirements based on the security level. The premise for this is that inherently the primary biometric identifiers for identifying the individual from the close-range face information and long-range gait information captured from video of a walking person, have several desirable properties under ideal and constrained operating environments, like universality, distinctiveness, permanence, collectability, acceptability, and resistance to spoofing. However, in reality, these systems operate in not so ideal environments. As a result, none of these traits can provide perfect recognition, and there is a need to improve the performance of these systems for day-to-day, civilian public access application scenarios. This is required for wide-spread diffusion and deployment of automated identification technologies based on non-intrusive, user friendly, biometric traits. Certain high level contextual information (soft characteristics) like gender, ethnicity, age, emotion/aggression, height, weight, and eye colour information could be extracted from the same video surveillance footage. Although, these soft characteristics are not unique and reliable, weak on their own, and are not capable of being decisive, they do provide important secondary level-additional demographic information about 
the user. They can certainly complement the identity information provided by the stronger biometric identifiers like face. The forensic identity recognition community have been using such soft characteristics for suspect and victim identification for a long time. The usage of secondary biometric identifiers - like the gender, age, ethnicity of the person, or the emotion- like aggression - on their own may not be useful to detect a criminal or identify an impostor. However, if used along with primary identifiers, it would be possible to enhance the robustness, reliability, and performance for different user and security requirements. Figure 3 shown below is the fusion structure for the combining primary and secondary identifier information

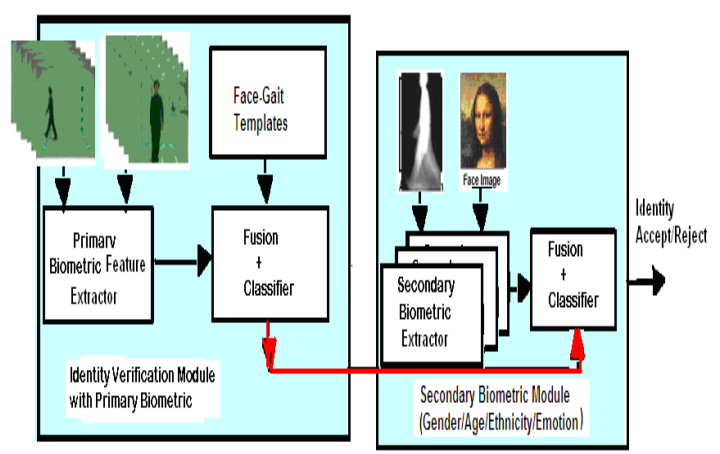

Fig. 6. Fusion structure for primary and secondary identifiers using face and gait patterns.

If the evidence from the primary biometric modules is not sufficient to take a decision about the identity of the speaker, the secondary biometric modules supplement the evidence and enhance the confidence level of the decision process. Such a setup for authentication process can provide several benefits. Modules in such authentication structures with primary biometric identifiers - gait image from long range camera 1 , face image from short range camera2, followed by gender, age and emotion extraction (secondary identifiers) will spring into action, increasing the level of security to meet the requirements of increasing authentication accuracy [3].

\section{CONCLUSIONS}

In this paper we have presented a review of current biometric identification technologies and suggested the potential of face and gait biometric traits for next generation biometric technologies. Some of work in progress in relation to development of face-gait fusion models, the importance of primary and secondary biometric traits and the role of fusion protocols in addressing the requirements of next generation biometrics is discussed. The future work will involve evaluation of fusion models being developed for different face-gait databases in terms of false accept rates, false reject rates and equal error rates under controlled and uncontrolled environments.

\section{REFERENCES}

[1] A.K. Jain, Next Generation Biometrics, Department of Computer Science \& Engineering, Michigan State University, Department of Brain \& Cognitive Engineering, Korea University, December 10, 2009

[2] I. K. Shlizerman, R. Basri, 3D Face Reconstruction from a Single Image Using a Single Reference Face Shape, IEEE TRANSACTIONS
ON PATTERN ANALYSIS AND MACHINE INTELLIGENCE, VOL. 33, NO. 2, FEBRUARY 2011

[3] S.M.E. Hossain, G. Chetty, Next Generation Biometric Identity Verification Based on Face- Gait Biometrics International Conference on Biomedical Engineering and Technology, Malaysia. Kuala Lumpur, June 17-19, 2011

[4] J. C. Vazquez, M. Lopez and P. Melin, Real Time Face Identification Using a Neural Network approach, soft comp. for regcogn, based on biometric, SCI 312, pp. 155- 169, @ Springer-Verlag Berlin, Heidelberg 2010

[5] F. Gaxiola, P. Melin and M. Lopez, Modular Neural Network for Person Authentication Using Counter Segmentation of the Human Iris Biometrics Measurement, Soft Comp. for Revogn, Based of Biometric, SCI 312, pp. 137-153, @ Springer-Verlag, Berlin Heidelberg 2010

[6] S. Berretti, A. Bimbo and P. Pala, 3D face recognaition using isogeodesic stripes, IEEE transaction on pattern analysis and machine intelligence, vol. 32, no. 12, December 2010

[7] Human Face Recognition, Advantages and disadvantages of 3D face recognition,

http://www.tutorial.freehost7.com/human_face_recognition /biometrics_and_human_biometrics.htm

[8] Fingerprint Identification Technology, Principles of fingerprint biometrics, www.biometricvision.com

[9] J. Feng, A.K. Jain, Fingerprint alteration, submitted to IEEE TIFS 2009.

[10] S. Bengio and J. Mariethoz, Biometric Person Authentication IS A Multiple Classifier Problem, Google Inc, Mountain View, CA, USA, bengio@google.com, IDIAP Research Institute, Martigny, Switzerland, marietho@idiap.ch

[11] Biometric technology, Hand Geometry Identification Technology, www.biometricvision.com

[12] L. Yuan, Z. Mu, and Z. Xu, Using Ear Biometrics for Personal Recognition, School of Information Engineering, Univ. of Science and Technology Beijing. Beijing 100083, yuanli64@hotmail.com

[13] Comparisons of Various Biometric Technologies, www.biometricvision.com

[14] M. N. Eshwarappa and M. V. Latte, Bimodal Biometric Person Authentication System Using Speech and Signature Features, International Journal of Biometrics and Bioinformatics, (IJBB), Volume (4): Issue (4)

[15] B. Son and Y. Lee, Biometric Authentication System Using Reduced Joint Feature Vector of Iris and Face, Division of Computer and Information Engineering, Yonsei University, 134 Shinchon-dong, Seodaemoon-gu, $\quad$ Seoul 120-749, Korea, \{sonjun,yblee\}@csai.yonsei.ac.kr. (T. Kanade, A. Jain, and N.K. Ratha (Eds.): AVBPA 2005, LNCS 3546, pp. 513-522, 2005.@ Springer-Verlag Berlin Heidelberg 2005)

[16] T. Bennett, New Iris Color Surgery, May 19, 2010, www.ehow.com

[17] N. B. Boodoo, R. Subramanian, Robust Multi- biometric Recognition Using Face and Ear Images, (IJCSIS) International Journal of Computer Science and Information Security, Vol. 6, No. 2, 2009

[18] F. Perronnin, J. C. Junqua, J. L. Dugelay,Biometrics Person Authentication: From Theory to Practice

[19] Emerging Biometrics, FBI Biometric Center for Excellence, http://www.biometriccoe.gov/Modalities/Emerging_Biometrics.htm

[20] Chakraborthy R., Rengamani H., Kumaraguru P., Rao R., "The UID Project": Lessons Learned from the Wast and Challenges Identified for India, Cyber Security, Cyber Crime and Cyber Forensics: Application and Perspective, Copyright (C) 2011, IGI Global.

[21] Pohsiang Tsai, Tich Phuoc Tran and Longbing Cao, A New Multimodal Biometric for Personal Identification, Faculty of Engineering and Information Technology - University of Technology, Sydney Australia, Pattern Recognition, Recent Advances, pp341-366

[22] A. P. Yazdanpanah, K. Faez, R. Amirfattahi, MULTIMODAL BIOMETRIC SYSTEM USING FACE, EAR AND GAIT BIOMETRICS, 10th International Conference on Information Science, Signal Processing and their Applications (ISSPA 2010), 978-1-4244-7167-6/101\$26.00 C2010 IEEE

[23] Schuldt Christian, Laptev Ivan and Caputo Barbara, "Recognizing Human Actions: A Local SVM Approach". In Proceedings ICPR 2004. ftp://ftp.nada.kth.se/CVAP/users/laptev/icpr04actions.pdf (retrieved on 28th May 2011).

[24] Schuckers M.E., "Bayesian Method"Computational Methods in Biometric Authentication: Statistical Method for performance evolution, (C) Springer-Verlag London Limited 2010.

[25] Cunningham P. and Delany S. J., k-Nearest Neighbour Classifiers, University College Dublin, Padraig.Cunningham@ucd.ie, Dublin 
Institute of Technology Sarahjane.Delany@comp.dit.ie , Technical Report UCD-CSI-2007-4, March 27, 2007.

[26] G. Shakhnarovich T. Darrell, On Probabilistic Combination of Face and Gait Cues for Identification, Artificial Intelligence Laboratory, Massachusetts Institute of Technology, 200 Technology Square, Cambridge MA 02139, fgregory,trevorg@ai.mit.edu

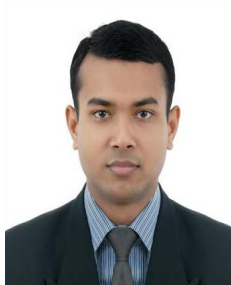

S. M. Emdad Hossain is a Post Graduate (Research) student in Faculty of Information Science and Engineering in University of Canberra. He also started his teaching in the same faculty. Prior to come to University of Canberra, he was doing Master of Information System in Central Queensland University, Australia. He also received Bachelor in Information Technology (Hons) from Multimedia University, Malaysia. He published number paper in International Conference and Journal. His research interests include biometric technology, computer security and pattern recognition.

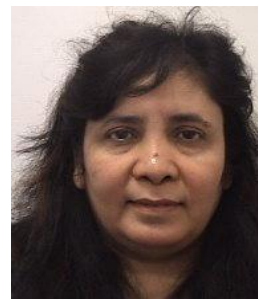

Girija Chetty, PhD is an Assistant Professor and Head of Software Engineering discipline in Faculty of Information Sciences and Engineering in University of Canberra, Australia. She received her Bachelors and Masters(Research) degrees in Electrical Engineering and Computer Science from India, and Doctorate in Information Sciences and Engineering from Australia. She has several years of research, teaching and industry experience from India and Australia, and has led several research, development and consulting projects in the related areas. She has published over 80 research papers in peer-reviewed International Journals and Conferences, and serves on editorial and review panels for several Journals and Conferences, including Biometrics, Multimedia Intelligence and Security, and Pattern Recognition and Computer Vision. Her research interests include biometrics, image and video coding, pattern recognition, computer vision and artificial intelligence. 